\title{
A low complexity multi-layered space frequency coding detection algorithm for MIMO- OFDM
}

\begin{abstract}
A low complexity multi-layered space frequency OFDM (MLSF-OFDM) coding scheme is presented with the proposed two detection algorithms, fast QR decomposition detection algorithm or denoted as FAST-QR and enhanced FAST-QR (E-FAST-QR). Both algorithms not only reduce the implementation complexity of $\mathrm{QR}$ decomposition but also show a good performance in terms of bit error rate (BER). Hence, the proposed detection algorithms can be used to maintain guaranteed quality of service (QoS) in MIMO-OFDM system.
\end{abstract}

Keyword: MIMO-OFDM; MLSF-OFDM; MLSTBC-OFDM; QR; SIC-ZF 